\title{
COMPREENDENDO A AUTOIMAGEM E A AUTOESTIMA EM ADOLESCENTES COM CÂNCER: UMA REVISÃO INTEGRATIVA
}

\section{REVISÃO INTEGRATIVA}

MORAIS, Tamires da Silva ${ }^{1}$

CARNIEL, Andreia Queiroz ${ }^{2}$

MORAIS, Tamires da Silva. CARNIEL, Andreia Queiroz. Compreendendo a autoimagem e a autoestima em adolescentes com câncer: Uma revisão integrativa. Revista Científica Multidisciplinar Núcleo do Conhecimento. Ano 05, Ed. 05, Vol. 10, pp. 125-140. Maio de 2020. ISSN: 2448-0959, Link de acesso: https://www.nucleodoconhecimento.com.br/psicologia/adolescentes-com-

\section{cancer}

\section{RESUMO}

O câncer é considerado uma doença degenerativa, consequente do acúmulo de lesões nas células que induz a reprodução desordenada das mesmas, dando origem aos tumores. Os efeitos colaterais do tratamento do câncer na adolescência podem comprometer a autoimagem e a autoestima e, repercutir na relação dos adolescentes consigo mesmo e com o outro. O objetivo desta revisão foi observar na literatura, os reflexos da doença na autoimagem e autoestima dos adolescentes. Os resultados apontaram que a autoimagem e a autoestima em adolescentes são afetadas com a manifestação da doença e seu tratamento. Portanto, considera-se relevante disponibilizar nos hospitais um espaço para escuta dos adolescentes, no intuito de criar possibilidades para minimizar o sofrimento e promover bem-estar psíquico.

${ }^{1}$ Graduação em Psicologia pela Faculdade Municipal Professor Franco Montoro (2018).

${ }^{2}$ Mestre em Ciências Médicas pela Universidade de Campinas- Unicamp. 
Palavras-chave: Câncer, adolescentes, autoimagem, autoestima.

\section{INTRODUÇÃO}

O câncer é caracterizado como uma doença degenerativa, consequente do acúmulo de lesões nas células que induz a reprodução anormal das mesmas, dando assim, origem aos tumores. Esta alteração celular possui dimensões graves que, ameaçam a vida do sujeito. Em alguns casos, a doença pode incidir por outras partes do organismo, ao qual se caracteriza como metástases (SOUZA; SANTOS, 2015).

Alguns autores como Esteves (2010) e Leite, Nogueira e Terra (2015), salientam que o câncer se difere dos outros tipos de doenças crônicas, pois há chances consideráveis de ocasionar mutilações, deformidades, dor e forte impacto psicológico.

A doença neoplásica (termo utilizado no meio médico para a definição do câncer), pode acometer indivíduos de qualquer faixa etária, independentemente de fatores culturais, econômicos ou sociais, ocorrendo na mesma proporção em ambos os sexos. Atualmente, o câncer é visto como um problema de saúde pública em inúmeros países. No Brasil, o câncer é uma das doenças com o maior índice de óbito, perdendo apenas para as doenças cardiovasculares (INCA, 2017).

Em 2015 o INCA divulgou a estimativa para o biênio 2016-2017, na qual conjecturouse o surgimento de aproximadamente 12.600 novos casos de câncer em crianças e adolescentes no país (INCA, 2015). Já em 2017 o INCA estimou que, para o biênio 2018-2019 ocorrerão 12.500 novos casos de câncer em crianças e adolescentes, posto que, a região Sudeste contemplará os maiores índices de novos casos, em torno de 5.300 (INCA, 2017).

No Brasil, o câncer corresponde à segunda causa de morte entre crianças e adolescentes em todas as regiões do país. Sob a ótica clínica, as neoplasias infantojuvenis normalmente apresentam menores períodos de latência, crescem mais rápido e são mais invasivas, contudo, respondem melhor ao tratamento que as diagnosticadas em adultos (INCA, 2017; REZENDE et al., 2009; IAMIN, 2011). 
Conforme os dados do INCA (2015) os tumores com maior incidência em crianças e adolescentes são: Neuroblastomas, Ritinoblastomas, Leucemias, Osteossarcomas, Sarcomas, Linfomas, Tumor de Wilms, Tumor Germinativo e os do Sistema Nervoso Central.

De acordo com a Organização Mundial da Saúde (OMS), a adolescência é uma etapa evolutiva que frequentemente tem início com as modificações físicas e se encerra com a inserção social, profissional e econômica do sujeito e com sua autonomia na sociedade. Portanto, a adolescência é um período de transição entre a infância e a vida adulta, cercada por transformações biológicas, emocionais, cognitivas e sociais, que não devem ser compreendidas indissociavelmente (MELO, 2006; LUCON, 2012).

Lucon (2012) afirma que as alterações corporais que acontecem nessa fase são universais, contudo, existem certas peculiaridades, tal como, as alterações psicológicas e de relações que se diversificam de uma cultura para outra, de um grupo para outro e até mesmo de um indivíduo para outro, dentro do mesmo grupo.

A aparência física para os adolescentes corresponde à percepção sobre a imagem que ele cria de si mesmo, e que serve como critério para ser apreciado pelos outros com quem se relaciona. Desta forma, as mudanças são percebidas por eles como parte de sua autoimagem levando a se valorizar ou, a sentir-se inferior. Assim, o corpo torna-se instrumento de resposta que os adolescentes procuram conduzir ao outro e a ele próprio (MELO, 2006).

Perina (2010) e Oliveira (2014) evidenciam que o tratamento do câncer em adolescentes depende da localização, do tipo histológico e do estadiamento. As modalidades terapêuticas utilizadas são: cirurgia, quimioterapia e radioterapia, combinadas ou não, o qual depende da avaliação médica sobre cada caso. $O$ tempo de tratamento varia entre seis meses a dois anos e meio.

Perina (2010) aponta que o diagnóstico de câncer para um adolescente acarreta inúmeras mudanças em seu contexto individual, familiar e social. Assim, se de um momento para o outro o jovem defrontar-se com o diagnóstico de câncer, todas as 
transformações causadas pelo processo do adolescer, somadas a sentimentos como incerteza e medo irão se intensificar a começar do instante em que o adolescente experiencia o adoecer (BULLA et al., 2015). Portanto, evidencia-se que vivenciar os processos de adolescer e adoecer é significativamente difícil e complexo.

O presente artigo, por meio da revisão integrativa, buscou compreender os reflexos que o câncer acarreta na autoimagem e na autoestima dos adolescentes, visto que, os efeitos colaterais do ponto de vista físico, emocional e social provocados pela doença e pelo tratamento podem causar alterações na autoimagem e trazer repercussões nas relações consigo mesmo e com o outro.

\section{METODOLOGIA}

Este estudo trata-se de uma revisão integrativa. O processo de tal revisão consistiu em três etapas: 1. Revisão de literatura (levantamento on-line em base de dados científicas da área das ciências médicas); 2. Avaliação crítica dos artigos; 3. Discussão dos conteúdos qualitativos a partir dos resultados dos artigos.

$\mathrm{Na}$ primeira etapa, revisão de literatura (levantamento on-line em base de dados científicas da área das ciências médicas): o levantamento foi realizado na Biblioteca Virtual de Saúde (BVS) que contém as seguintes bases de dados: Scielo (Scientific Electronic Library Online), PEPSIC (Periódicos Eletrônicos em Psicologia), Lilacs (Literatura Latino-Americana e do Caribe em Ciências da Saúde), PubMed (Biblioteca Nacional de Medicina Americana) e Medline (Medical Literature Analysis and Retrieval System Online).

Como estratégia de busca, utilizou-se os seguintes termos descritores: "câncer; adolescentes; autoimagem; autoestima" e "câncer; adolescents; self-image; selfEsteem" no período cronológico entre maio de 2018 a março de 2016. O recorte temporal foi dos últimos doze anos e, nos idiomas: espanhol, inglês e português.

Como critério de inclusão encontra-se apenas artigos, teses, dissertações, monografia e documentos do Ministério da Saúde (INCA) correlacionados ao tema e que 
colaboram com o objetivo apresentado, selecionou-se artigos de revisão e de pesquisas qualitativas, bem como, teses, dissertações e monografias que tivessem como tema principal a autoimagem e/ou autoestima de adolescente com câncer e os estudos que falassem sobre a vivência e sentimentos do adolescente com câncer. Foram excluídas publicações indisponíveis na íntegra para acesso na internet e que não se correlacionasse com o tema principal.

$\mathrm{Na}$ etapa de avaliação crítica das publicações, os estudos selecionados foram lidos na integra e em sua língua de origem, fichados, analisados e avaliados conforme a metodologia proposta nos mesmos. Os temas comuns às publicações foram reagrupados em temas específicos surgindo categorias de análise de temas comuns, contemplando assim, a autoimagem e autoestima em adolescentes com câncer, para melhor compreensão dos mesmos.

E, na etapa de discussão dos conteúdos qualitativos a partir dos resultados das publicações, após a coleta dos dados, conforme o proposto realizou-se a interpretação dos índices coletados das buscas bibliográficas. Esta é uma forma de conhecimento prévio científico, sobre o que o mundo acadêmico já tem de conhecimento do tema pesquisado, assim como, de possíveis contribuições para pesquisas nos estudos qualitativos.

\section{RESULTADOS}

Tendo em vista a problemática deste trabalho e a aplicação dos critérios de inclusão e exclusão, vinte e sete publicações (27), sendo que, dezoito (18) consistem em artigos científicos, três (03) dissertações, duas (02) teses, dois (02) documentos do Ministério da Saúde e uma (01) monografia foram obtidas como amostra para compor tal estudo.

A princípio atentou-se à apresentação dos resumos dos estudos, sendo que a maior preocupação dos autores foi evidenciar parte do conteúdo do texto abordado na introdução, metodologia, itens dos resultados, discussão e considerações finais, deixando a desejar quanto aos novos direcionamentos. O resumo é de significativa 
relevância para os artigos, haja vista que, introduz o leitor ao texto, convidando-o para a leitura do artigo na íntegra.

Pode-se observar similaridade dos temas abordados entre as publicações avaliadas, possibilitando assim uma análise dos dados apresentados nestas publicações, reagrupando em grupos (categorizações) para discussão do material coletado. Desta forma, obtiveram-se os seguintes resultados:

\section{A MANIFESTAÇÃO DA DOENÇA NA ADOLESCÊNCIA}

A preparação para a vida adulta requer um ajustamento do jovem frente às inúmeras transformações sucedidas, contudo, de todas as adequações pressupõe-se que a mais complexa seja o ajustamento à transição saúde-doença, dado que, o adolescente além de encarar e necessitar adequar-se as transformações da adolescência, também precisa ajustar-se a "ser adolescente-doente" (IAMIN, 2011).

As implicações acarretadas pelo diagnóstico na vida de um adolescente são profundas. Sua vida é desorganizada, debilitada e o adolescente precisa de uma nova estruturação de seus pensamentos, comportamentos e uma nova rotina. O adoecimento crônico de adolescentes possui uma condição singular devido à etapa transitória que se encontram no desenvolvimento (REZENDE, 2011). O impacto da doença sobre o desenvolvimento físico e psicológico do adolescente pode ser observado durante as diversas fases do tratamento, a revelação do diagnóstico é uma das fases mais críticas, normalmente a doença é percebida como uma tragédia e é fator responsável pela desestruturação psíquica, essa afecção ocupa um espaço de temor, com o papel de uma doença cruel e avassaladora que ainda é tida como uma "sentença de morte" (MELO, 2006). Segundo Leite, Nogueira e Terra (2015) a experiência do diagnóstico e do tratamento é peculiar para cada sujeito, contudo, temores, aflições, preocupações e anseios acompanham os pacientes desde 0 diagnóstico até o fim do tratamento. 


\section{OS ASPECTOS PSICOSSOCIAIS EM ADOLESCENTES COM CÂNCER}

Os autores Melo (2006) e Souza e Santos (2015) destacam que o adolescente acometido pelo câncer tem as mesmas necessidades que um adolescente em estado sadio, porém, essas necessidades tendem a ser prejudicadas pelo processo de tratamento. A autonomia cobiçada na adolescência tende a ser afetada devido ao processo de tratamento longo e as hospitalizações frequentes e, se faz necessária grande dependência de seus cuidadores. Os adolescentes são privados de atividades sociais, como ir a festas, competições esportivas, lugares públicos ou realizar qualquer atividade que represente risco, por isso, é comum que decorrente às restrições do tratamento haja alterações de humor e de comportamento. O estabelecimento da autonomia e da independência é fundamental para a formação da identidade, visto que, esses fatores ficam comprometidos pelas restrições já mencionadas, a doença pode influenciar negativamente a formação da identidade. Melo cita em sua dissertação de mestrado uma pesquisa realizada em adolescentes com doenças crônicas que constatou que o impacto do câncer sobre a imagem corporal, o processo de escolarização e as relações familiares interferia mais que qualquer outra afecção crônica na adolescência.

O adolescente com câncer ao ser distanciado do contexto escolar sofre não apenas uma perda educacional, tal como, uma perda de relacionamento social, uma vez que, o distanciamento dos colegas provoca um sentimento de ser esquecido, levando a uma sensação de solidão e isso interfere em sua vida particular (IAMIN, 2011; GARCÍA; GÓMEZ-MAQUEO, 2016). Pini, Gardner e Hugh-Jones (2013) ainda alertam que o planejamento para educação superior e carreira pode ser igualmente abalado por um diagnóstico de câncer na adolescência.

Lucon (2012) evidencia que para o adolescente a vontade de frequentar o ambiente escolar é rodeada de significações que a escola possui para ele, visto que, tal ambiente simboliza uma aceitação social assegurada pelas ideologias consolidadas pela sociedade de que na escola transitam os alunos saudáveis. Riel et al. (2014) 
assinala que a escola exerce um papel relevante no funcionamento social dos jovens. Para Pini, Gardner e Hugh-Jones (2013) a reintegração na escola posteriormente a um tempo de ausência pode ser um desafio importante para os adolescentes. Em seu estudo, tais autores concluíram que as maiores taxas de ausências da escola estavam associadas com experiências mais desfavorecidas de reintegração, levantaram questões sobre o temor de ser aceito novamente em seu grupo de pares, os adolescentes revelaram também a preocupação sobre ser provocados ou intimidados por sua aparência devido à alopecia e perda de peso.

De acordo com a literatura mencionada pelas autoras Cicogna, Nascimento e Lima (2010) a ausência capilar tem consequências psicossociais, haja vista que, o cabelo é significativo na comunicação social e sexual, sendo símbolo político, cultural e social. A alopecia vem sendo correlacionada à perda de identidade e de atratividade. Desta forma, as atitudes dos pacientes no que diz respeito à perda do cabelo podem diversificar conforme a relevância que dão ao cabelo, preparação e informações recebidas, nível de perda e mecanismos somáticos e psíquicos. Constantemente é no período de realização da quimioterapia e, fundamentalmente, a partir da manifestação de seus efeitos colaterais que o adolescente se percebe de fato doente. Vieira et al. (2012) cita que a alopecia no indivíduo que faz tratamento quimioterápico o caracteriza como paciente oncológico entre os sujeitos a sua volta.

\section{A AUTOIMAGEM E AUTOESTIMA EM ADOLESCENTES COM CÂNCER}

Os diversos tipos de tratamento acarretam inúmeros efeitos colaterais que afetam a autoimagem e a sexualidade, prejudicando as relações interpessoais dos jovens. Os adolescentes expressam imensa angústia no tocante à alopecia, à amputação de membros, à alteração da massa corporal, ao medo de esterilidade e às modificações ginecológicas. Tais implicações levam a uma transformação na imagem corporal do adolescente e pode afetar o desenvolvimento de uma autoimagem coerente. O adolescente pode se sentir diferente dos outros jovens e, transformado em relação à visão que tinha de seu corpo antes do tratamento, o que lhe provoca estranheza 
(REZENDE, 2011; LUCON, 2012; DOCHERTY et al., 2015; VIEIRA et al., 2012; SOUZA; SANTOS, 2015).

Cicogna, Nascimento e Lima (2010) indicam que o preconceito gera desconforto, uma vez que, muitos indivíduos tendem a se distanciar daqueles que estão fora dos "padrões de normalidade", comportamento este que pode exacerbar os sentimentos de isolamento vivenciados por adolescentes com câncer.

A quimioterapia manifestou-se como pertinente causa de sofrimento, em razão de causar modificação da autoimagem e temor da morte, para muitos adolescentes a queda de cabelo é a alteração mais acentuada no corpo e está vinculada à sua autoestima. Os jovens, com a alteração da aparência, sentem-se longe dos "padrões de normalidade", visto que, a ausência de cabelo deixa evidenciar uma imagem que não é saudável. A compreensão do preconceito do outro perturba e pode ocasionar danos emocionais (BULLA et al., 2015).

A pesquisa de Oliveira (2014) constatou que a alopecia é o aspecto mais aludido pelos jovens, dado que, a queda de cabelo associa-se aos efeitos colaterais provocados pela quimioterapia. Assim, o adolescente se sente diferente, o que gera um sentimento de dor, que poderá acarretar um isolamento social. Os jovens no início do tratamento não admitem a queda de cabelo por resultar em uma mudança visual imensa na imagem que será identificada pelas outras pessoas e, deste modo, sentemse incomodados com sua aparência.

Em seu trabalho, Fan e Eisen (2009) citaram estudos que revelaram que a autoestima estava negativamente associada às alterações na aparência. Três estudos qualitativos evidenciaram que a diminuição da autoestima foi relacionada ao distúrbio de imagem corporal. As mudanças na aparência física colaboraram para uma sensação de ser diferente de colegas saudáveis e para um sentimento de "Pareço feio ou doente".

Cicogna, Nascimento e Lima (2010) em sua pesquisa observaram que o efeito adverso é o fator mais aludido pelos adolescentes, sendo as alterações físicas as mais 
mencionadas, em virtude de sua relação com a autoimagem e com a integridade de todo o corpo. Para os jovens, os efeitos colaterais mais predominantes são náuseas e vômitos, alteração de peso, indisposição, mal-estar, falta de apetite, manchas na pele, dor de cabeça e alopecia. No tocante à imagem corporal, os participantes da pesquisa ressaltaram, fundamentalmente, aspectos associados à alopecia e à alteração de peso. O emagrecimento, mais comum no início, além de perturbar os adolescentes se torna mais acentuado a partir dos comentários de outros indivíduos, comentários esses que atribuem ao emagrecimento uma interpretação de desgaste devido ao tratamento. São mudanças que influenciam a autoimagem de adolescentes e possibilitam que outros sujeitos percebam que algo está ocorrendo e afetando o seu corpo.

Em seu estudo, Oliveira (2014) acrescenta que várias adolescentes se compararam com sua autoimagem antes do câncer e, dessa forma, disseram se sentir feias e fora do padrão de beleza admitido pela sociedade e, tal percepção provoca alterações psíquicas e sociais pertinentes em suas vidas. O trabalho de Pinquart (2013) comprovou que as meninas tendem a ter mais insatisfação corporal do que meninos. Tal fato pode gerar maiores efeitos negativos das doenças crônicas na autoimagem das meninas como consequência do maior destaque depositado na aparência feminina pela sociedade.

\section{DISCUSSÃO}

A aparência física é essencial para a cultura juvenil e é o fator que mais colabora para os níveis de autoestima em adolescentes (WILLIAMSON et al., 2010). Na adolescência o autovalor torna-se um aspecto essencial, o adolescente passa a atribuir maior importância a percepção e opinião que os outros têm sobre si. A menina tende a apresentar mais conflitos durante a adolescência e o desenvolvimento da autoimagem, pois considera mais as opiniões que os outros têm em relação a ela (SBICIGO; BANDEIRA; DELL' AGLIO, 2010).

lamin (2011) destaca que diante de episódios de transição do desenvolvimento e de saúde-doença acontece desestruturação, ansiedade, irritabilidade, estresse, 
depressão, alterações na autoestima e alterações no autoconceito. Para os jovens esta sobreposição de transições demanda aceitar novos papéis na sociedade, na escola, na família, sendo uma etapa relevante da construção de sua identidade. Diante do adoecimento, a identidade dos jovens com câncer também sofre modificações, uma vez que, as expectativas do próprio adolescente, da família, da sociedade, os projetos e os planos sofrem uma interrupção com o adoecimento e permanecem em suspenso. Forma-se então um abismo entre o que imaginava que seria capaz de fazer e o que de fato é capaz a partir do adoecimento.

Kim, White e Patterson (2016) consideram que a adolescência é a fase onde são tomadas as decisões de vida mais relevantes e múltiplos papéis sociais são cumpridos, por isto, é provável que vivenciar tal período associado a um processo de adoecimento pode causar um sofrimento emocional intenso e, a sensação de incapacidade de desempenhar papéis sociais pode se tornar fonte de culpa.

Os autores Brito et al. (2008) corroboram que as manifestações físicas e psíquicas do câncer modificam para cada indivíduo e modificam no mesmo sujeito em momentos distintos. Cada um experiencia e encara sua doença de maneira diferente, conforme sua história de vida, o relacionamento com os outros, as vantagens e desvantagens de estar doente, a capacidade de tolerância à insatisfação.

Se para um adolescente saudável já há uma discrepância entre a imagem idealizada e a imagem real de seu corpo em metamorfose, para um jovem doente essas diferenças acentuam-se em virtude de que, adicionado a tais diferenças próprias desta fase, se percebe diante de uma transformação visível imposta pela doença, assim sendo, os jovens necessitam elaborar o luto do corpo ideal e saudável e se apoderar de um corpo debilitado (CAZAROLLI et al., 2011).

É pertinente salientar que atrelado a elaboração do luto do corpo e da identidade infantil, da relação com os pais da infância e de alguns desejos, tais "mortes" são fundamentais para o próprio desenvolvimento, o adolescente necessita elaborar também o luto do corpo saudável e se apoderar de um corpo adoecido, para conseguir aceitar a doença e lidar com a realidade imposta por ela. Associado a esses lutos 
importantes, está a doença correlacionada a uma "sentença de morte", que vem carregada de incertezas, medos e angústias que influencia a maneira como o adolescente enfrentará seu adoecimento.

Os efeitos negativos visíveis da doença na imagem corporal podem ser mais intensos na adolescência do que na infância devido às reações negativas de outros adolescentes no tocante a atratividade física e seu ajustamento como amigo ou parceiro (PINQUART, 2013). Contudo, o apoio social pode amenizar o impacto dos efeitos colaterais do câncer e de seu tratamento sobre as mudanças na autoimagem dos adolescentes. Esse suporte pode vir da família e amigos, tal como, da equipe médica (FAN; EISER, 2009).

\section{CONSIDERAÇÕES FINAIS}

Conforme a literatura, na adolescência vivencia-se uma turbulência de transformações biológicas, emocionais e sociais e, vivenciar o adolescer associado a um processo de adoecimento, geralmente repercute na autoimagem do indivíduo, na relação consigo mesmo e com o outro e, causa sofrimento emocional intenso. As vivências de uma rotina radicalmente transformada e as exigências do tratamento colocam 0 adolescente em situações de conflito, seja com ele mesmo, seja com a circunstância a qual está submetido.

Portanto, sugere-se a necessidade de os profissionais de saúde conhecer as repercussões da doença, do processo quimioterápico e suas inferências para o adolescente, principalmente sobre a correlação entre o câncer e a autoimagem em pacientes jovens. Por esta razão, é importante haver um espaço para escuta dos adolescentes, a fim de criar possibilidades para minimizar o sofrimento, promover bem-estar psíquico, elaboração de experiências e sentimentos, visando assim, à reorganização de aspectos emocionais. 


\section{REFERÊNCIAS}

BRITO, A. O. et al. Respostas aos eventos vivenciados pelos adolescentes portadores de câncer. Journal of Nursing UFPE on line, v. 2, n. 3, p. 263-269, jul.-set. 2008. Disponível em: <https://periodicos.ufpe.br/revistas/revistaenfermagem/>. Acesso em: 27 de dezembro de 2017.

BULLA, M. L. et al. O mundo do adolescente após a revelação do diagnóstico de câncer. Revista Mineira de Enfermagem, v. 19, n. 3, p. 681-688, jul-set. 2015. Disponível em: <http://www.reme.org.br/artigo/detalhes/1032>. Acesso em: 26 de dezembro de 2017.

CAZAROLLI, E. et al. Sentimentos de adolescentes com câncer: um estudo qualitativo. Revista Contexto \& Saúde, v. 11, n. 20, p. 1365-1369, jan.jun. 2013. Disponível em: $<$ https://revistas.unijui.edu.br/index.php/contextoesaude/article/view/1810>. Acesso em: 04 de janeiro de 2018.

CICOGNA, E. C.; NASCIMENTO, L. C; LIMA, R. A. G. Crianças e adolescentes com câncer: experiências com a quimioterapia. Revista Latino-Americana de Enfermagem, v. 18, n. 5, set.-out. 2010. Disponível em: $<$ http://www.redalyc.org/html/2814/281421935005/>. Acesso em: 28 de dezembro de 2017.

DOCHERTY, S. L. et al. The adolescent and young adult with cancer: a developmental life course perspective. In: Seminars in oncology nursing. WB Saunders, v.31, n. 3, p. 186-196, ago. 2015. Disponível em: <http://www.sciencedirect.com/science/article/pii/S0749208115000480>. Acesso em: 03 de janeiro de 2018.

ESTEVES, A. V. F. Compreendendo a criança e o adolescente com câncer em tratamento quimioterápico durante a utilização do brinquedo. 2010. $179 \mathrm{f}$. Tese (Doutorado em Psicologia) - Faculdade de Filosofia, Ciências e Letras de Ribeirão 
Preto, Universidade de São Paulo, Ribeirão Preto, SP, 2010. Disponível em: <http://www.ffclrp.usp.br/imagens_defesas/27_05_2011_15_39_45_61.pdf>. Acesso em: 01 de março de 2016.

FAN, S.-Y.; EISER, C. Body image of children and adolescents with cancer: a systematic review. Body image, v. 6, n. 4, p. 247-256, 2009. Disponível em: <http://www.sciencedirect.com/science/article/pii/S174014450900062X>. Acesso em: 02 de janeiro de 2018.

GARCÍA, A. A. G.; GÓMEZ-MAQUEO, M. E. L. Estilo de afrontamiento y calidad de vida en adolescentes con cáncer. Gaceta Mexicana de Oncología, v. 15, n. 1, p. 39, 2016.

Disponível

em:

<http://www.sciencedirect.com/science/article/pii/S1665920116000079>. Acesso em: 03 de janeiro de 2018.

IAMIN, S. R. Adolescentes na vivência do câncer: a interface da pesquisacuidado e acompanhamento terapêutico. 2011. 208 f. Dissertação (Mestrado em Biotecnologia Aplicada à Saúde da Criança e do Adolescente) - Faculdade Pequeno Príncipe, Curitiba, PR, 2011. Disponível em: <http://www.focodh.com.br/files/Dissertacao-Mestrado---doc-completo27082011.pdf>. Acesso em: 23 de dezembro de 2017.

INCA - Instituto Nacional de Câncer. Estimativa 2016: incidência de câncer no Brasil. Rio de Janeiro, RJ: INCA, 2015. Disponível em: $<$ http://www2.inca.gov.br/wps/wcm/connect/tiposdecancer/site/home/infantil>. Acesso em: 09 de novembro de 2016.

INCA - Instituto Nacional de Câncer. Estimativa 2018: incidência de câncer no Brasil. Rio de Janeiro, RJ: INCA, 2017. Disponível em: $<$ http://www.inca.gov.br/estimativa/2018/estimativa-2018.pdf>. Acesso em: 15 de maio de 2018

KIM, B.; WHITE, K.; PATTERSON, P. Understanding the experiences of adolescents and young adults with cancer: a meta-synthesis. European Journal of Oncology 
Nursing, v. 24, p. 39-53, 2016. Disponível em: <http://www.sciencedirect.com/science/article/pii/S1462388916300515>. Acesso em: 02 de janeiro de 2018.

LEITE, M. A. C.; NOGUEIRA, D. A.; TERRA, F. S. Avaliação da autoestima em pacientes oncológicos submetidos a tratamento quimioterápico. Revista Latino Americana de Enfermagem. Ribeirão Preto, v. 23, n. 6, p. 1082-1089, nov.-dez. 2015. Disponível em: <http://www.scielo.br/pdf/rlae/v23n6/pt_0104-1169-rlae-23-0601082.pdf >. Acesso em: 01 de março de 2016.

LUCON, C. B. O impacto do câncer na adolescência: contribuições da classe hospitalar no processo de enfrentamento. Conhecimento \& Diversidade. Niterói, v. 4, $\quad$ n. $7, \quad$ p. 55-70, 2012. Disponível em: $<$ https://ebooks.unilasalle.edu.br/index.php/conhecimento_diversidade/article/view/62 3>. Acesso em: 05 de janeiro de 2018.

MELO, C. S. C. A. Perfil e preocupações de adolescentes em tratamento de câncer atendidos em unidades pediátricas. 2006. 129 f. Dissertação (Mestrado em Psicologia) - Universidade de Brasília, Brasília, DF, 2006. Disponível em: <http://repositorio.unb.br/bitstream/10482/2965/1/2006_Carmem\%20Sophia\%20Cam pelo\%20de\%20Albuquerque\%20e\%20Melo.pdf>. Acesso em: 01 de março de 2016.

MENEZES, C. N. B. et al. Câncer infantil: organização familiar e doença. Mal-Estar e Subjetividade. Fortaleza, v.7, n.1, p. 191-210, mar. 2007. Disponível em: <http://pepsic.bvsalud.org/pdf/malestar/v7n1/11.pdf>. Acesso em: 05 de março de 2016.

OLIVEIRA, K. C. X. Criança, adolescente e família frente ao diagnóstico com câncer. 2014. 76 f. Trabalho de Conclusão de Curso (Monografia) - Curso de Enfermagem, Faculdade Católica Salesiana do Espírito Santo, Vitória, ES, 2014. Disponível em: <http://www.ucv.edu.br/fotos/files/TCC_2014-2_Kelly.pdf>. Acesso em: 05 de janeiro de 2018. 
PERINA, E. M. Qualidade de vida de adolescentes sobreviventes de câncer na infância e sua relação com ansiedade, depressão e estresse pós-traumático. 2010. 156 f. Tese (Doutorado em Saúde da Criança e do Adolescente) - Faculdade de Ciências Médicas, Universidade Estadual de Campinas, Campinas, SP. 2010. Disponível em: <http://repositorio.unicamp.br/handle/REPOSIP/312079>. Acesso em: 02 de janeiro de 2018.

PINI, S.; GARDNER, P.; HUGH-JONES, S. The impact of a cancer diagnosis on the education engagement of teenagers-patient and staff perspective. European Journal of Oncology Nursing, v. 17, n. 3, p. 317-323, 2013. Disponível em: <http://www.sciencedirect.com/science/article/pii/S1462388912000853>. Acesso em: 03 de janeiro de 2018.

PINQUART, M. Body image of children and adolescents with chronic illness: a metaanalytic comparison with healthy peers. Body image, v. 10, n. 2, p. 141-148, 2013. Disponível em: <http://www.sciencedirect.com/science/article/pii/S1740144512001374>. Acesso em: 28 de dezembro de 2017.

REZENDE, A. M. Compreendendo o adolescente com câncer: vivências da doença. 2011. 92 f. Dissertação (Mestrado em Saúde Coletiva) - Programa de Pós Graduação em Ciências da Saúde do Centro de Pesquisas René Rachou, Belo Horizonte, MG, 2011. Disponível em: <https://www.arca.fiocruz.br/handle/icict/6244>. Acesso em: 02 de janeiro de 2018.

REZENDE, A. M. et al. Vivências de crianças e adolescentes com câncer: o desenho fala. Iniciação Cientifica Cesumar. Maringá, v. 11, n. 1, p. 73-82, jan.-jun. 2009. Disponível

em: <http://periodicos.unicesumar.edu.br/index.php/iccesumar/article/view/600file://C:/Us ers/hardware/Downloads/600-2855-2-PB.pdf>. Acesso em: 02 de março de 2016.

RIEL, C. A. H. P. et al. Self-perception and quality of life in adolescents during treatment for a primary malignant bone tumour. European Journal of Oncology 
Nursing, v. 18, n. $3, \quad$ p. 267-272, 2014. Disponível em: <http://www.sciencedirect.com/science/article/pii/S1462388914000143>. Acesso em: 02 de janeiro de 2018.

SBICIGO, J. B.; BANDEIRA, D. R.; DELL' AGLIO, D. D. Escala de Autoestima de Rosenberg (EAR): validade fatorial e consistência interna. Psico - USF. Itatiba, v. 15, n. 3, p. 395-403, set.-dez. 2010. Disponível em: $<$ http://www.scielo.br/pdf/pusf/v15n3/v15n3a12.pdf>. Acesso em: 02 de março de 2016.

SOUZA, D. S. M.; SANTOS, L. G. O adolescente com câncer: o adoecimento potencializando os conflitos da adolescência. Revista Cientefico. Fortaleza, v. 15, n. 30, p. 61-78, jan.jun. $2015 . \quad$ Disponível em: $<$ https://revistacientefico.devrybrasil.edu.br/cientefico/article/view/108>. Acesso em: 28 de dezembro de 2017.

VIEIRA, G. B. et al. Impacto do câncer na autoimagem do indivíduo: uma revisão integrativa. Revista Baiana de Enfermagem. Salvador, v. 26, n. 2, p. 533-540, mai.ago. $2012 . \quad$ Disponível em: <https://search.proquest.com/openview/ff2e2fd756cc2f662df7ce32b2ce9587/1 ?pqorigsite $=$ gscholar\&cbl=2040112 $>$. Acesso em: 03 de janeiro de 2018 .

WILLIAMSON, $\mathrm{H}$. et al. Adolescents' and parents' experiences of managing the psychosocial impact of appearance change during cancer treatment. Journal of Pediatric Oncology Nursing, v. 27, n. 3, p. 168-175, fev. 2010. Disponível em: $<$ http://journals.sagepub.com/doi/abs/10.1177/1043454209357923>. Acesso em: 02 de janeiro de 2018.

Enviado: Fevereiro, 2019.

Aprovado: Maio, 2020. 